\title{
Computing Labeled Orthogonal Drawings
}

\author{
Carla Binucci, Walter Didimo, Giuseppe Liotta, and Maddalena Nonato \\ Università di Perugia \\ \{binucci, didimo, liotta, nonato\}@diei.unipg.it
}

\begin{abstract}
This paper studies the problem of computing labeled orthogonal drawings. A label is modeled as a rectangle of prescribed size and it can be associated with either a vertex or an edge. Several optimization goals are taken into account. Namely, the labeled drawing can be required to have minimum total edge length, minimum width, minimum height, or minimum area. We present ILP models to compute optimal drawings with respect to the first three objectives and an algorithm exploiting these models which computes a drawing of minimum area (the compaction problem is known to be NP-complete in general).
\end{abstract}

\section{Introduction}

The increasing demand of visualization technologies to display maps and schemas with textual or multimedia content associated with vertices and/or edges has been motivating a significant research effort towards designing algorithms and systems that display labeled drawings of graphs. Among the application domains where these technologies are relevant we mention Cybergeography (Web maps and Internet maps), Software Engineering (UML schemas), and Information Systems (data-base schemas, organization charts). (See also [5] for more application domains).

At a first sight, one might think that computing a labeled drawing of a graph is "yet-another-version" of the well-known labeling placement problem that has attracted a lot of research in the computational geometry and cartography communities (see, e.g. [7, 10,13,14] and the on-line bibliography of Strijk and Wolff [17]). Namely, one possible approach for solving the problem is that of removing the labels, computing a drawing, and putting back the labels. However, this approach has several drawbacks in practice. For example, most graph drawing algorithms and systems draw all vertices as boxes having the same (usually quite small) size: if labels have to be drawn inside vertices (such as in a UML schema), a global re-scaling of the drawing may be required at each label insertion. Further, if the graph to display is large (such as a portion of the Web graph), then most graph drawing systems would try to draw groups of edges close with each other so to avoid unnecessary wastes of space on the computer screen. However, this strategy may contrast with the necessity of adding large textual labels on the edges in a post-processing phase while maintaining readability and compactness of the diagram.

On the other hand, the graph drawing labeling problem allows more flexibility than the labeling placement problem. While in the latter problem the geometry of 
the map to be labeled is fixed and given as part of the input, in the graph drawing context the geometry is part of the output. This observation has suggested a radically different approach to the problem, based on computing a drawing of the input graph by taking into account also the space needed for adding the labels [2:411].

This paper studies the problem of computing labeled orthogonal drawings. A label is modeled as a rectangle of prescribed size and it can be associated with either a vertex or an edge. Several optimization goals are taken into account. The labeled drawing can be required to have minimum total edge length, minimum width, minimum height, or minimum area. Previous papers on this subject include the seminal paper by Klau and Mutzel [11, the first to present an ILP model for orthogonal drawings of minimum total edge length with labeled vertices, and the work in 2] where several heuristics for orthogonal drawings of minimum area with labeled edges are presented. Both problems of computing orthogonal drawings of minimum area and minimum total edge length are NPcomplete, even when edges and vertices are not labeled [16].

The main contributions of this paper are as follows.

- An ILP model for orthogonal drawings with labels both on edges and on vertices is presented. Given a labeled orthogonal representation $H$, the ILP model can be used to compute a labeled orthogonal drawing of $H$ with minimum total edge length. Variants of the model compute a drawing of minimum width or of minimum height. We remark that the model in this paper is the first ILP model for edge labeling of orthogonal representations. Concerning vertex labeling, our model is different from the one proposed by Klau and Mutzel [1] in which a vertex $v$ is mapped to a point and a label of $v$ is drawn as a box whose perimeter includes $v$. In our model the label of $v$ is also a box, but $v$ can be either a point on the perimeter or a point inside the box. Our choice is motivated by applications in which vertices are identified with labels, such as drawing UML diagrams and ER-diagrams.

- We describe a strategy for computing a labeled orthogonal drawing with minimum area among those preserving a given orthogonal representation. Our approach is based on computing several solutions of the ILP model for width minimization under a certain constraint on the height of the drawing. Our solution strategy is markedly different from standard integer quadratic programming techniques, which appear to be not as effective as our method for handling the specific problem of area minimization. We experimentally observe that the number of ILP solutions required by our algorithm is quite small ( 5 on the average) and that this number does not seem to depend on the number of vertices of the input graph.

- As a special case, our algorithm for area minimization can be used for unlabeled drawings. As far as we know, no other algorithm is known in the literature to this aim, except for some specific classes of orthogonal representations [3].

For reasons of space several details are omitted in this abstract. A more complete version including extensive experimental analysis and heuristics for labeled drawings is available at 1 . 


\section{Labeled Orthogonal Drawings}

We assume familiarity with graph theory 6], graph planarity [15, and graph drawing [59]. Let $G=(V, E)$ be a 4-planar graph. An orthogonal drawing of $G$ is a drawing of $G$ such that each vertex of $G$ is mapped to a distinct point of the plane and each edge of $G$ is drawn as a sequence of horizontal and vertical segments between its end-vertices. An orthogonal grid drawing of $G$ is an orthogonal drawing such that vertices and bends are mapped to points of the integer grid. An orthogonal representation of $G$ is an equivalence class of planar orthogonal drawings of $G$, such that: (i) For each edge $(u, v)$ of $G$ all drawings in the class have the same sequence of left and right turns (bends) along $(u, v)$, while moving from $u$ to $v$. (ii) For each vertex $v$ of $G$, and for each pair $\left\{e_{1}, e_{2}\right\}$ of clockwise consecutive edges incident on $v$, all drawings in the class determine the same angle between $e_{1}$ and $e_{2}$.

Let $L=\{\lambda\}$ be a set of rectangular labels of given integer width $w(\lambda)$ and given integer height $h(\lambda)$. We assume that each $\lambda$ is associated with one edge of $G$ and no two labels are associated with the same edge. Each $\lambda$ is an edge-label. Let $N=\{\nu\}$ be a set of rectangular labels of given integer width $w(\nu)$ and given integer height $h(\nu)$ such that each $\nu$ is associated with one vertex of $G$ and such that no two labels are associated with the same vertex. Each $\nu$ is a vertex-label. In the following we shall use the term "label" instead of vertex-label or edge-label when the context does not give rise to ambiguities.

Consider the triplet $(G, L, N)$. A labeled orthogonal drawing $\Gamma$ of $(G, L, N)$ is a drawing such that: (i) $G$ is represented as a planar orthogonal drawing and (ii) each label in $L \cup N$ is represented as a rectangle.

The following definitions characterize a good labeling.

Definition 1. An edge $e \in \Gamma$ with associated an edge-label $\lambda \in L$ is properly labeled if:

(EL1) $\lambda$ is a rectangle in $\Gamma$ with height $h(\lambda)$ and width $w(\lambda)$

(EL2) $\lambda$ has only one side $s$ contained in one segment $i$ of $e$, and $s$ is properly contained in $i$. We say that $\lambda$ is drawn on $i$.

Note that (EL2) implies that there is an intersection between $\lambda$ and the segment on which $\lambda$ is drawn.

Definition 2. A vertex $v \in V$ with associated a vertex-label $\nu \in N$ is properly labeled if:

(VL1) $\nu$ is a rectangle in $\Gamma$ with height $h(\nu)$ and width $w(\nu)$

(VL2) $v$ is a point either on the perimeter or in the interior of $\nu$. We say that $\nu$ is drawn on $v$.

Note that (VL2) implies that there is an intersection between $\nu$ and each segment incident on the vertex associated with $\nu$.

Definition 3. A labeled drawing $\Gamma$ is properly labeled if: 
(EL) Each edge of $\Gamma$ is properly labeled

(VL) Each vertex of $\Gamma$ is properly labeled

(NI) There is no overlap between a label and any other element of the drawing, except for the intersections implied by labels drawn on edges or vertices.

\section{An ILP Model for Labeled Orthogonal Drawings}

Let $G$ be a 4-planar graph and let $H_{G}$ be an orthogonal representation of $G$. Let $L$ and $N$ be a set of labels for the edges and for the vertices of $G$ respectively. In this section we present an ILP model for computing a properly labeled orthogonal grid drawing $\Gamma$ of $(G, L, N)$ of minimum total edge length among all drawings with shape defined by $H_{G}$. The model can be modified to minimize either the width or the height of the drawing; it can also be used as a basic component for an algorithm that computes a drawing of minimum area among those whose shape is defined by $H_{G}$ (see Section 4).

In this abstract we only provide the mathematical foundation and the main ideas behind our model. We first introduce the notation that will be used in the model (Subsection 3.1); then we show a characterization of properly labeled orthogonal drawings and describe the idea behind the ILP model (Subsection 3.2).

\subsection{Notation}

We need a mathematical formalism for translating the conditions given by Definitions 1, 2] and 3 in the ILP model. We introduce first some notation for segments of $\Gamma$ representing edges of $G$. Then we focus on labels and consider relations between labels, vertices and edges. The notation is similar to that introduced in previous works on the same topic 11,12.

Let $\Gamma$ be a (not necessarily properly) labeled orthogonal drawing of $(G, L, N)$. Let $X^{h}=\left\{\chi^{h}\right\}$ and $X^{v}=\left\{\chi^{v}\right\}$ be the set of the maximal horizontal and vertical chains of edge segments of $\Gamma$, respectively. Note that a chain may consist of a single point which we treat as a zero-length segment. Let $S^{h}$ be the union of the segments of all the maximal horizontal chains, and let $S^{v}$ be the union of the segments of all the maximal vertical chains. Let $S=S^{h} \cup S^{v}$. We enumerate the edge segments of $\Gamma$ by associating each element $i$ of $S$ with a distinct positive integer number, called index of $i$. We denote by $\operatorname{first}\left(\chi^{h}\right)$ and $\operatorname{first}\left(\chi^{v}\right)$ the segment of minimal index in $\chi^{h}$ and $\chi^{v}$, respectively. Given a horizontal (resp. vertical) segment $i$ of $\Gamma$, we denote by $C(i)$ its y-coordinate (resp. x-coordinate). Each non-zero length segment $i \in S$ is bounded by two maximal chains orthogonal to $i$ denoted by $\sup (i)$ and $\inf (i)$ with $C(\operatorname{first}(\sup (i)))>C(\operatorname{first}(\inf (i)))$. The length of a segment $i$ is given by $C(\operatorname{first}(\sup (i)))-C(\operatorname{first}(\inf (i)))$. Each vertex $u \in V$ belongs to a unique maximal horizontal chain, $\chi^{h}(u)$ and to a unique maximal vertical chain, $\chi^{v}(u)$. Let $C_{x}(u)=C\left(\operatorname{first}\left(\chi^{v}(u)\right)\right)$ and $C_{y}(u)=C\left(\operatorname{first}\left(\chi^{h}(u)\right)\right)$ denote the x-coordinate and the y-coordinate of vertex $u$, respectively. 
Let $\lambda \in L$ be an edge-label associated with an edge $e$ of $G$. Label $\lambda$ can be drawn on any of the segments representing e. $S(\lambda)$ denotes the set of segments of $e$ and $S(L)$ is the set of all candidate segments for edge-labels, i.e. $S(L)=$ $\cup_{\lambda \in L} S(\lambda)$. We say that $\lambda$ overlaps a segment $i \in S(\lambda)$ if the intersection between $i$ and one of the two sides of $\lambda$ parallel to $i$ is not empty. Note that if $\lambda$ is drawn on $i$ then $\lambda$ overlaps $i$ (see (EL2) in Definition 1). However, if $\lambda$ overlaps $i$ but the drawing is not properly labeled, then $\lambda$ may be not drawn on $i$.

For each edge-label $\lambda$ and for each edge segment $i \in S(\lambda)$, we define a 4 tuple $\Sigma^{i, \lambda}=\left\{\sigma_{o}^{i, \lambda}, \sigma_{O}^{i, \lambda}, \sigma_{p}^{i, \lambda}, \sigma_{P}^{i, \lambda}\right\}$ of segments. Roughly, the elements of $\Sigma^{i, \lambda}$ describe the position of the sides of the rectangle representing $\lambda$ with respect to $i$. The elements of $\Sigma^{i, \lambda}$ are defined as follows (in our notation $o, O$ stand for "orthogonal" and $p, P$ stand for "parallel"):

- if $\lambda$ overlaps $i$, then $\sigma_{o}^{i, \lambda}$ and $\sigma_{O}^{i, \lambda}$ are the sides of $\lambda$ orthogonal to $i ; \sigma_{p}^{i, \lambda}$ and $\sigma_{P}^{i, \lambda}$ are the sides of $\lambda$ parallel to $i$. Also, $C\left(\sigma_{O}^{i, \lambda}\right)>C\left(\sigma_{o}^{i, \lambda}\right)$ and $C\left(\sigma_{P}^{i, \lambda}\right)>$ $C\left(\sigma_{p}^{i, \lambda}\right)$.

- if $\lambda$ does not overlap $i$, then $\sigma_{o}^{i, \lambda}=\sigma_{O}^{i, \lambda}=\sigma_{p}^{i, \lambda}=\sigma_{P}^{i, \lambda}$ are zero-length segments, $C\left(\sigma_{P}^{i, \lambda}\right)=C\left(\sigma_{p}^{i, \lambda}\right)=C(i)$, and $C\left(\sigma_{O}^{i, \lambda}\right)=C\left(\sigma_{o}^{i, \lambda}\right)=C(f i r s t(\sup (i)))$.

We also define $\Sigma^{L}=\underset{i \in S(L)}{\cup} \Sigma^{i, \lambda}$.

Similar definitions are given for vertex-labels. Given a vertex $v \in \Gamma$ and its vertex-label $\nu \in N$, let $S(\nu)$ be the set of edge segments incident on $v$. Let $\Sigma^{\nu}=\left\{\sigma_{o}^{\nu}, \sigma_{O}^{\nu}, \sigma_{p}^{\nu}, \sigma_{P}^{\nu}\right\}$ be the 4-tuple of label segments introduced to represent $\nu$ on $\Gamma$, according to the following convention: $\sigma_{o}^{\nu}$ and $\sigma_{O}^{\nu}$ are vertical; $\sigma_{p}^{\nu}$ and $\sigma_{P}^{\nu}$ are horizontal; $C\left(\sigma_{O}^{\nu}\right)>C\left(\sigma_{o}^{\nu}\right)$ and $C\left(\sigma_{P}^{\nu}\right)>C\left(\sigma_{p}^{\nu}\right)$. We define $\Sigma^{N}=\cup_{\nu \in N} \Sigma^{\nu}$.

Finally, we need a notation for defining notions like "top", "bottom", "left" and "right" to express constraints that avoid intersection between our geometric objects. For an edge-label $\lambda \in L$, we define a 4-tuple $t(\lambda), b(\lambda), l(\lambda), r(\lambda)$ where $t(\lambda)$ is the top-most horizontal side of the rectangle representing $\lambda, b(\lambda)$ is the bottom-most, $l(\lambda)$ is the left-most vertical side, and $r(\lambda)$ is the right-most. Similar definition for a vertex-label $\nu \in N$ and corresponding $t(\nu), b(\nu), l(\nu), r(\nu)$. For a segment $i \in S$, if $i \in S^{h}$ then $t(i)=b(i)=i, l(i)=\inf (i), r(i)=\sup (i)$; else, if $i \in S^{v}$ then $t(i)=\sup (i), b(i)=\inf (i), l(i)=r(i)=i$.

\subsection{The ILP Model}

We provide six properties which translate the definition of properly labeled orthogonal grid drawing by using the notation of Subsection 3.1.

The constraints of the ILP model translate each property into a set of linear inequalities and integrality constraints. We use the notion of signed distance $\delta$. For each ordered pair of parallel segments $(i, j)$ in $S \cup \Sigma^{L} \cup \Sigma^{N}$ let $\delta(i, j)=$ $C(i)-C(j)$. Note that, $\delta(i, j)=-\delta(j, i)$.

The six properties are as follows.

(PLD1) For each $\lambda \in L$, there exists exactly one triplet $(\lambda, i, z)(\lambda \in L, i \in$ $S(\lambda), z \in\{p, P\})$ such that: 
- If $i \in S(\lambda) \cap S^{v}$ then $\left|\delta\left(i, \sigma_{z}^{i, \lambda}\right)\right|=w(\lambda)$ and $\delta\left(\sigma_{O}^{i, \lambda}, \sigma_{o}^{i, \lambda}\right)=h(\lambda)$.

- If $i \in S(\lambda) \cap S^{h}$ then $\left|\delta\left(i, \sigma_{z}^{i, \lambda}\right)\right|=h(\lambda)$ and $\delta\left(\sigma_{O}^{i, \lambda}, \sigma_{o}^{i, \lambda}\right)=w(\lambda)$.

We call such a triplet the position of $\lambda$.

(PLD2) If $i$ is the segment of the position of $\lambda$, then $C(\operatorname{first}(\sup (i)))>C\left(\sigma_{O}^{i, \lambda}\right)$ and $C($ first $(\inf (i)))<C\left(\sigma_{o}^{i, \lambda}\right)$.

Also, $\forall i \in S, C(f \operatorname{irst}(\sup (i)))-C(\operatorname{first}(\inf (i)))>0$.

(PLD3) Let $\nu \in N$ be the label associated with vertex $v \in V$, then $\delta\left(\sigma_{O}^{\nu}, \sigma_{o}^{\nu}\right)=$ $w(\nu)$ and $\delta\left(\sigma_{P}^{\nu}, \sigma_{p}^{\nu}\right)=h(\nu)$.

(PLD4) Let $\nu \in N$ be the label associated with vertex $v \in V$, then $0 \leq C_{x}(v)-$ $C\left(\sigma_{o}^{\nu}\right) \leq w(\nu)$ e $0 \leq C_{y}(v)-C\left(\sigma_{p}^{\nu}\right) \leq h(\nu)$.

(PLD5) Let $\alpha, \beta$ be two elements of $S \cup L \cup N$. At least one of the following conditions is verified:

(1) $\delta(b(\beta), t(\alpha))>0 .(2) \delta(l(\beta), r(\alpha))>0 .(3) \delta(b(\alpha), t(\beta))>0 .(4)$ $\delta(l(\alpha), r(\beta))>0$. (5) $\alpha$ is an edge segment and $\beta$ is a label drawn on $\alpha$.

(6) $\alpha$ is an edge segment incident on a vertex $v$, and $\beta$ is the label associated with $v$.

(PLD6) All vertices and bends have integer coordinates.

Theorem 1. Let $G$ be a 4-planar graph, $L$ be a set of labels for the edges of $G$, and $N$ be a set of labels for the vertices of $G$. A labeled orthogonal grid drawing $\Gamma$ of $(G, L, N)$ is properly labeled if and only if Properties (PLD1) - (PLD6) hold.

Based on Theorem [ an ILP model that minimizes the total edge length has the following objective function Min $\sum_{i \in S} C(f i r s t(\sup (i)))-C(f \operatorname{irst}(\inf (i)))$ and about fifty groups of constraints which implement Properties (PLD1) (PLD6) (for details see the manuscript at [1]).

In the implementation of the ILP problem we can omit the integrality constraints on the coordinates of vertices and bends, which improves the efficiency of the computations. With a technique similar to that presented in 12, it can be proved that the solutions of the resulting MILP problem are always grid drawings. The total number of variables and inequalities of the ILP model is $O\left((n+b)^{2}\right)$ where $n$ and $b$ are the number of vertices and bends of $\Gamma$, respectively.

We remark that it is easy to modify the model in order to minimize the width or the height of the drawing. We denote by $w_{\Gamma}$ and $h_{\Gamma}$ the width and the height of the bounding box of $\Gamma$, expressed in terms of grid points. The model for width minimization is obtained by using the objective function $\operatorname{Min}\left(w_{\Gamma}\right)$ and by adding the following constraints:

$$
\begin{gathered}
h_{\max }>C(i) \quad h_{\min } \leq C(i) \quad \forall i \in S^{h}, \\
w_{\max }>C(i) \quad w_{\min } \leq C(i) \quad \forall i \in S^{v}, \\
h_{\Gamma}=h_{\max }-h_{\min }, w_{\Gamma}=w_{\max }-w_{\min } .
\end{gathered}
$$




\section{Optimizing the Area}

In order to compute a labeled drawing of minimum area we exploit the ILP model of the previous section. Namely we set up a new objective function: $\operatorname{Min}\left(w_{\Gamma}+\right.$ $\frac{1}{M_{h}} \cdot h_{\Gamma}$ ) and add a new constraint $h_{\Gamma}<h_{m}$ where $M_{h}$ is an upper bound of the height of the drawing with optimal area. This corresponds to computing a drawing $\Gamma$ with height no greater than $M_{h}+1$ with minimum height among all drawings of minimum width. The algorithm iteratively searches for a new drawing with the same properties as $\Gamma$ and lower height. The algorithm stops when the search fails.

The idea relies on the fact that the minimum area drawing belongs to the set of Pareto optimal solutions of the multiobjective function $\operatorname{Min}\left[\begin{array}{l}w_{\Gamma} \\ h_{\Gamma}\end{array}\right]$, where $\Gamma$ is a properly labeled orthogonal grid drawing. Our method returns a subset of this set which the optimal solution belongs to.

In the following pseudo-code, Min-Width $\left(h_{m}\right)$ is a subroutine that either returns a properly labeled orthogonal grid drawing with minimum width constrained to $h_{m}$, if any, or it returns null.

Algorithm OptArea

Input: A triplet $(G, L, N)$ and an orthogonal representation $H_{G}$

Output: A properly labeled orthogonal grid drawing $\Gamma^{o p t}$ of $(G, L, N)$ with minimum area within $H_{G}$

begin

$h_{m}=M_{h}+1$

let $\Gamma=\Gamma^{o p t}=\operatorname{Min}-\operatorname{Width}\left(h_{m}\right)$

$h_{m}=h_{\Gamma}$

while $(\Gamma$ is not null $)$

let $\Gamma=\operatorname{Min}-\operatorname{Width}\left(h_{m}\right)$

if $(\Gamma$ is not null) then

if $\left(w_{\Gamma^{o p t}} \cdot h_{\Gamma^{o p t}}>w_{\Gamma} \cdot h_{\Gamma}\right)$ then $\Gamma^{o p t}=\Gamma$

$$
h_{m}=h_{\Gamma}
$$

end

Theorem 2. Let $G$ be a 4-planar graph and let $H_{G}$ be an orthogonal representation of $G$. Let $L$ and $N$ be a set of labels for the edges and for the vertices of $G$ respectively. Algorithm OptArea computes a properly labeled orthogonal grid drawing of $(G, L, N)$ with minimum area within $H_{G}$.

Since the widths of the drawings iteratively computed by the algorithm are increasing, $h_{m}$ can be updated to $\left(w_{\Gamma}^{o p t} \cdot h_{\Gamma}^{o p t} / w_{\Gamma}\right)$ instead of $h_{m}=h_{\Gamma}$. We experimentally observed that in most cases the number of algorithm iterations reduces significantly. Moreover, the number of ILP solutions required by our algorithm is quite small (5 in the average) and this number does not seem to depend on the number of vertices of the input graph. 


\section{Open Problems}

There are several open problems that we plan to investigate in the near future. Some of them are: (i) Refine our ILP model by valid inequalities in order to speed up the solution process. (ii) Extend the ILP model to graphs with high degree vertices. (iii) Design new heuristics for the graph drawing labeling problem.

\section{References}

1. C. Binucci, W. Didimo, G. Liotta, and M. Nonato. Computing labeled orthogonal drawings, manuscript: http://www.diei.unipg.it/PAG_PERS/binucci/binucci.htm.

2. C. Binucci, W. Didimo, G. Liotta, and M. Nonato. Labeling heuristics for orthogonal drawings. In Symposium on Graph Drawing (GD'01), volume 2265 of LNCS, pages 139-153, 2002.

3. S. Bridgeman, G. D. Battista, W. Didimo, G. Liotta, R. Tamassia, and L. Vismara. Turn-regularity and optimal area drawings of orthogonal representations. Computational Geometry: Theory and Applications, 16:53-93, 2000.

4. R. Castello, R. Milli, and I. Tollis. An algorithmic framework for visualizing statecharts. In Symposium on Graph Drawing (GD'00), volume 1984 of LNCS, pages 139-149, 2001.

5. G. Di Battista, P. Eades, R. Tamassia, and I. G. Tollis. Graph Drawing. Prentice Hall, Upper Saddle River, NJ, 1999.

6. F. Harary. Graph Theory. Addison-Wesley, Reading, MA, 1972.

7. K. G. Kakoulis and I. G. Tollis. An algorithm for labeling edges of hierarchical drawings. In Symposium on Graph Drawing (GD'97), volume 1353 of LNCS, pages 169-180, 1998.

8. K. G. Kakoulis and I. G. Tollis. On the complexity of the edge label placement problem. Computational Geometry: Theory and Applications, 18:1-17, 2001.

9. M. Kaufmann and D. Wagner. Drawing Graphs. Springer Verlag, 2001.

10. G. Klau and P. Mutzel. Optimal labeling of point features in rectangular labeling models. Mathematical Programming, Series B, To appear.

11. G. Klau and P. Mutzel. Combining graph labeling and compaction. In Symposium on Graph Drawing (GD'99), LNCS, pages 27-37, 1999.

12. G. Klau and P. Mutzel. Optimal compaction of orthogonal grid drawings. In Integer Programming and Combinatorial Optimization (IPCO'99), volume 1610 of LNCS, pages 304-319, 1999.

13. G. Klau and P. Mutzel. Optimal labelling of point features in the sliding model. In In Proc. COCOON'00, volume 1858 of LNCS, pages 340-350, 2001.

14. S. Nakano, T. Nishizeki, T. Tokuyama, and S. Watanabe. Labeling points with rectangles of various shape. In Symposium on Graph Drawing (GD'00), volume 1984 of $L N C S$, pages 91-102, 2001.

15. T. Nishizeki and N. Chiba. Planar Graphs: Theory and Algorithms, volume 32 of Annals of Discrete Mathematics. North-Holland, 1988.

16. M. Patrignani. On the complexity of orthogonal compaction. Computational Geometry: Theory and Applications, 19:47-67, 2001.

17. T. Strijk and A. Wolff. The map labeling bibliography. on-line: http://www.mathinf.uni-greifswald.de/map-labeling/bibliography/. 